ORL 1997;59:345-346

\title{
Subject Index Vol. 59, 1997
}

Acoustic neurinoma 193

neuroma $67,159,272$

trauma 303 Adenoid 286

Advanced otosclerosis 332 Allergic rhinitis 36 Amyloidosis 60 Anatomy 277 Anemia 73

Apoptosis 303 Arthritis 215 Ary-epiglottic folds 182 Aspartate 131 Aspiration 100 ATP 170

Auditory brainstem response 141,272

- $\quad$ polyp 336

Auricular chondritis 215

Autoantibody 10

Benign paroxysmal positional vertigo 155,

269 Benzalkonium chloride 39 Bilateral pseudo-internuclear ophthalmo-

plegia 122 Bone defect 277 Branchial arch syndrome 147

Calcium incorporation 4 Castleman's disease 339 Cell extrusion 303 Central compensation 326

conduction time 141 Cephalometric analysis 286 Cerebrospinal fluid leakage 115 Cervical

esophagus 119

lymph node enlargement 176

mass 339 Cochlear implant 23,230

nerve 67

Computer-assisted image analysis 258 Continuous interleaved sampling 23 CYFRA21-1 106

Cytomegalovirus 97

Destruction nystagmus 326 Differential staining 147 Disability 209 Dissociated nystagmus 122

Eccentric rotation 198 Elderly subjects 141

Electrically evoked auditory brainstem

response 67 Electromyography 159 Electron probe microanalysis 187 Electron-microscopic

immunocytochem-

istry 131 Endolymph 311 Endolymphatic hydrops 84, 292

- $\quad$ sac 127,311

Enzyme immunoassay 235

Epstein-Barr virus 97

Etretinate 147

Eustachian tube 166

Experimental hydrops 127

Facial morphology 286

nerve 277

- paralysis 159 Fluorochrome 4 Furosemide 248

Gerbil 243

Glomangioma 179

Glutamate 131

Glyceroltest 127 
Guinea pig 4,84,187,263,311,317

Haircell(s) 303,317 Head and neck squamous cell carcinoma 106

- - surgery 100 Hearing disorder 79 Histamine 50

challenge 39

Histiocytic necrotizing lymphadenitis 176 Histopathology 91 Hyperbaric oxygenation 79

Hyposmia 36

Immunocytochemistry 317 Immunoelectron microscopy 317 Immunohistochemistry 258,311

Impairment of adduction 122 Inhalation exposure 91 Inner ear 258,263

- disease 10

hair cells 131 Internal auditory canal 18 Intracellular free calcium 322

- sodium 322 Intracranial recording 272 Ion transport 170

Iron 73 Isoforms 311

Kikuchi's disease 176 KTP/532 laser 45

Labyrinthectomy 209 Larynx 182,235

Laser Doppler flowmetry 50, 280 Liberatory maneuver 155 Lymph node hyperplasia 339

Macula utriculi 193

Malformation 147

Mediastinal tumor 296

Ménière’s disease 10, 84, 135, 209, 292

Mental state 326

Microdissection 147

Middle ear 64,147

- $\quad$ - epithelium 170

Minor salivary gland 60

Mondini malformation 115

Multiple sclerosis 122

Myasthenia gravis 122

Nasal cavity 179

mucosa 50,280

obstruction 36 Neurilemmoma 182 Neurinoma 182 Neurofibroma 182 Neurofilaments 258

Neurotologic surgery 18 NF-H 258

NF-L 258

NF-M 258

Nitric oxide synthase 311

Non-recurrent laryngeal nerve 57

Obstructive sleep apnea 286 Occult carcinoma 296 Ocular counterrolling 198 Olfaction 36

Olfactory mucosa 91 Organ of Corti 243 Organotypic culture 243 Oropharyngeal dysphagia 100

Osmolality 292 Osteoma 64 Otitis media 170 Otoacoustic emissions 73 Otoconia 4, 187,263

KA I $\backslash G$ E I $\quad$ (C) 1997 S. Karger AG,

E-Mail karger@karger.ch Fax + 41613061234 http://www. karger. ch

345

Otosclerosis 277 Outer hair cell 322 Oxymetazoline 39

Particle repositioning maneuver 155

Pendular rotation test 269

Perchloroethylene gas 91 
Perilymphatic pressure 135

Perineurioma 336

Polychromatic labeling 4

Poorly differentiated component 296

Postembedding immunolabeling 317

Posterior semicircular canal 269

Pregnancy 292

Presbycusis 141

Pressure treatment 84

Prognosis 159

Promontory 64

Purinoceptors 170

Repair 303

Repeated caloric tests 326 Rhinitis medicamentosa 39 Rhinostereometry 39, 50, 280 Roll 198

Saccadic hypermetria 122 Salivary gland tumours 97 Scanning electron microscopy 115,166,

263

Schwannoma 182 Sjögren's syndrome 60 Snoring 45 Sodium transport 170 Speech coding 23

- $\quad$ understanding 23

Spindle cell tumor 336

Squamous cell papilloma 119

Stapedectomy 332

Stapes malformation 115 Sternocleidomastoid muscle-clavicle

myoosseous flap 238 Streptomycin 209, 263 Stria vascularis 248 Strial edema 248 Swallowing

therapy 100 Synaptophysin 317

T cell receptor 215 TATI 106

Tectorial membrane 127 Temporal bone 18,147

- $\quad$ - pathology 230

Teratogenesis 147

Three-dimensional reconstruction 193 Thyroid carcinoma 238

gland 296

surgery 57 Tinnitus 79 Tongue 60

Tonsil 286 Tracheal plasty 238

- $\quad$ stenosis 296

Translation 198

Transmission electron microscope 193 Treatment 292 Tumor markers 106 Tympanic bulla 166

- $\quad$ membrane displacement 135

Tympanitis 215

Type II collagen 215

Uvulopalatoplasty 45

Vacuolation, marginal cells 248 Varicella zoster virus 235 Vasoconstrictors 39 Vertigo 209

Vesicles, intermediate cells 248 Vestibular organ 127 Video nystagmography 198

Videoendoscopic biofeedback 100 Vocal cord palsy 235

Western blot assay 10 Wistarrat 166

346

ORLVol. 59, 1997 
Subject Index 